\title{
Enhancing General Contractors Capacities With Collaborative Networks
}

Amirhossein Mohebifar

Ph.D. candidate for Project Management and Construction, Tarbiat Modares

University, Tehran, Iran

a.mohebifar@modares.ac.ir
DOI 10.5592/otmcj.2013.2.3

Research paper

\section{Keywords}

General Contractor,

Subcontratctor, Collaborative Network, Communication, Large Projects, Coordination
Mohammad Hossein Sobhiyah

Ph.D. in Project Management and

Construction, Tarbiat Modares University, Iran
Samira Haghbin

Master in Project Management and

Construction, Shahid Beheshti

University, Tehran, Iran

THIS RESEARCH IS MOTIVATED BY CONSIDERING NEW REQUIREMENTS OF GENERAL CONTRACTORS (GC), RESULTED FROM MODERN DAY BUSINESS ENVIRONMENT AND CAPABILITIES OF COLLABORATIVE NeTWORKS (CN) TO MEET THESE REQUIREMENTS. GC should establish, manage and maintain an efficient network of suppliers and subcontractors to achieve complex construction project's goals and guarantee the project performance.

In recent years, complication and specialization of large projects of construction industry has led into wide recruitment of specialists contractors and, therefore, dividing projects into small segments.

Thus, there is more emphasis on maintaining coordination between various sections and contractors, which will in turn guarantee the overall performance of a project. This paper will look at advantages of establishing $\mathrm{CN}$ to enhance capability and meet requirements of GC in Construction Projects. Concepts related to other knowledge areas such as Collaborative Networks are introduced, too. It is hoped that this will help in further research on developing the concept of $\mathrm{CN}$ in construction project management. The paper discusses similarities between $\mathrm{GC}$ and $\mathrm{CN}$ and possibility of establishing a coordinating part for $\mathrm{GC}$, in the format of a virtual enterprise. The findings imply that most of the GC needs for becoming international and successful could be covered by Collaborative Networks' specifications. 


\section{INTRODUCTION}

In this conceptual paper, development methodology for two research fields of General Contractor (GC) and Collaborative Networks (CN) is considered and similarities existing between requirements of $\mathrm{GC}$ and advantages of $\mathrm{CN}$ are studied. High level of complication in big construction projects, caused by presence of various subcontractors and suppliers, forces GC to focus on coordination with subcontractors. Rapid changes in technology often make it necessary for firms to depend on external technological knowledge and skills, as well as their internal resources. Nowadays, many firms rely extensively on external linkages to acquire new technological knowledge and use strategies such as technology licensing and collaborative agreements for this purpose. Inter-firm collaboration is an important vehicle for creation of technological competencies (Schoenmakers \& Duysters, 2006). In addition, GC is needed to make coordination possible and maintain the network of suppliers and subcontractors in a hierarchical structure determined though contractual relationships. Many researchers argued that construction project organization should consider an interdependent aspect of the cooperative work structure (Chinowsky, et al., 2008) but none of them has been studied two fields of GC and CN together. Therefore, this paper attempts to review both fields literature to find similarities in their specifications.

\section{Literature Review}

\section{Definition of General Contractor}

General Contractor is a contractor, who is responsible for preparing, coordinating and completing all tasks of one project. (ISO)

Recently, it is understood that the success of big projects in construction industry depends heavily on contractor-subcontractor relationship. Therefore, there has been more emphasis both on inter-organizational coordination between GC and subcontractors and search for new concepts to improve this relationship based on experiences of different construction firms.

Coordinating subcontracted work that is more sophisticated, requires more time and incurs higher costs as well .The cost affects performance, coordination and inspection level. How these variables are affected by coordination cost can serve as a criterion for selecting relationship type i.e. in comparison with a competitive relationship, in a partnership relationship the $\mathrm{GC}$ is required to put in more effort to manage subcontracted work (Lee, et al., 2009).

The management system of general contracting and subcontracting is widely applied in international construction market. The qualification of construction enterprises consists of the general contracting management, the specialty subcontracting and labor service (Fan \& Meng-jun, 2008).

Time, cost, quality and resources, having mutual effect, are four major control items for any General Contractor in construction project. The best solution is to achieve high quality with low cost in a proper time limit, use up resources if possible and keep them balanced as well.

In construction projects, along with increase in the number of participants, including the owner, designer, general contractor, subcontractors and resource vendors, uncertainty will also increase. It is suggested that coordination is an effective method to minimize the impact of uncertainty. Some even believe that GC can lead the coordination of implementation (Shuquan \& Kongguo, 2008).

GCs are large companies with great abilities and experiences in construction that have great knowledge of specialty sub-contractors and suppliers and vendors. They choose proper subcontractors and suppliers for project based on project type and requirements and try to coordinate them and lead them until the objectives of project have been achieved. GC and suppliers behave as parts of a network that relate with kinds of contract relations or strategic partnership.

Project implementation team can be seen as a virtual enterprise, which constitutes the supply-chain in a project carrier through collaboration work in response to rapid changes in construction market. This is visible in cooperation existing between some parts of project teams with different responsibilities. However, in order to get market advantages at fierce competition, the GC must find the best suppliers to build up highly efficient supply chain in project management. The relationship between General Contractor and suppliers is a cooperative and efficient one in supply (Xiaolin, et al., 2008).

\section{Collaborative Networks}

The notion of "network" is nowadays a central issue in many fields including social sciences, communications, computer science, physics, and even biology and ecosystems (Dorogovtsev, et al., 2003). A large variety of collaborative networks have emerged during the last years as a result of the challenges faced by both the business and scientific worlds (Camarinha-Matos \& Afsarmanesh, 2005).

It is a common assumption that participation in Collaborative Network has the potential of bringing benefits to the involved entities. These benefits include an increase in "survivability" of organizations in market turbulence, as well as the possibility of achieving common goals by excelling individual capabilities. On the basis of these expectations we can find, among others, the following factors acquisition of a larger dimension, access to new/wider markets and knowledge, sharing risks and resources, joining complementary skills and capacities which allows each entity to focus on 
its core competencies while keeping a high level of agility etc Although everybody has an intuitive notion of what collaboration is about, this concept is often confused with cooperation. For many people the two terms are indistinguishable. The ambiguities reach a higher level when other related terms are considered, such as networking, communication, and coordination (CamarinhaMatos \& Afsarmanesh, 2006).

In today's industry, CN manifest in a large variety of forms. Moving from the classical supply- chains format, characterized by relatively stable networks with well-defined roles and requiring only minimal coordination and information exchange, more dynamic structures are emerging in industry. Some of these organizational forms are goal-oriented, i.e. focused on a single project or business opportunity, such as the case of virtual enterprises (VE) (Camarinha-Matos, et al., 2009).

A CN is a network consisting of a variety of entities (e.g. organizations and people) that are largely autonomous, geographically distributed, and heterogeneous in terms of their operating environment, culture, society, capital and goals, but that collaborates to better achieving common or compatible goals, thus jointly generating value. Additionally, their internal interactions are supported by a computer network (Camarinha-Matos, et al., 2009).

Virtual organization of CN frequently demands for information and communication technology to support coordination of cross-organizational business process chains. Service-oriented software technologies provide promising means to regulate and enforce coordination of cross-organizational software service interactions but miss organizational abstractions and methodology (Zippiness \& Emmerich, 2008).

\section{Structure and Characteristics of General Contractors}

The concept of GC has been under completion over half a century in western countries. During the said time, a certain number of technology-advanced, large-scaled, internationalized GC has been formed. All of the large international GC implements globalization strategy and their business scope is wide. The SNC LAVALIN Company in Canada, with 40 years' experience in international engineering contract market, has commenced business in more than 100 countries. The Bechtel Company located in USA has successfully achieved over 20,000 projects in more than 140 countries and regions on all continents; its business area ranges from civil engineering to the oil, chemical, metallurgy, railway, telecommunication, industry and civil construction project, transportation, electric power, water conservancy and other infrastructure projects etc

Research and analysis indicated the following as common characteristics of these enterprises: (Wen \& Xiao, 2010)

- Globalization of scope and diversification of business

- Organizational structure of enterprise adapted to general contract

$\checkmark$ Advanced project management technique

> Powerful finance capacity

Technology innovation

\section{Necessities of General Contractors in Construction Projects}

Five serious challenges faced by constructors and suppliers in construction field, relying on past experience and foreseeable in $\mathbf{2 0 2 0}$ are as follows: (Camarinha-Matos, et al., 2009)

- Simultaneous process and task doing capability

- Large increase in human resource performance and human satisfaction

- Immediate conversion of documented information obtained from various extended sources of data to useful knowledge for effective decision making

- Enjoying enough flexibility to respond to necessary changes and opportunities of market in last minute

Innovation in providing construction process with concentration on decreasing dimensions' scales

- Also other challenges and necessities of these contractors which have been recognized in past researches could be read in following:

- High financial Competence (Ballard \& Kim, 2005), (Wen \& Xiao, 2010) (Xiaolin, et al., 2008).

- Long-term alliance- collaborative maintains (PARK \& HAN, 2012), (Han, et al., 2010).

- Rapid communication, information exchange and knowledge exchange flow (PARK \& HAN, 2012), (Tserng \& Yin, 2010).

These challenges need new organizational structures, business models, theories, processes and technologies to prepare companies for addressing modern day changes in business environment. To overcome the constraints, these companies need cooperation with one another.

\section{Structures and Characteristics of Collaborative Networks}

Amongst $\mathrm{CN}$ some networks are goaloriented in which intense collaboration (towards a common goal) is practiced between their partners, as opposed to longer term strategic alliances, where in fact not collaboration but cooperation is practiced by members.

Goal-oriented networks can be either driven by continuous production/service provision activities or the common aim of grasping a single (collaboration) opportunity. In Goal-oriented networks, the first case of $\mathrm{CN}$ labeled as Continuous-Production driven, includes those networks that have a long-term duration and a relatively stable situation during that time, with a clear definition of members' roles along the value chain (Camarinha-Matos \& Afsarmanesh, 2006).

How will an organization benefit from participating in $\mathrm{CN}$ ? Will the benefits compensate for the extra overhead, loss 
of some control and even taking the risks that collaboration implies? These are the main questions that many small and medium-sized enterprise (SME) managers ask when the issue of collaboration is brought up. In fact, effective collaboration involves considerable preparation costs / time, in addition to the operational overheads and risks, which represent barriers to the rapid formation of dynamic coalitions in response to business opportunities. As a basic rule, in order to support rapid formation of $\mathrm{CNs}$, it is necessary that potential partners are ready in advance and prepared to participate in such collaboration. This readiness includes compliance with common interoperable infrastructure, operating rules and collaboration agreement, amongst others. Any collaboration also requires a base level of trust among organizations. Therefore, the concept of Virtual Organization (VO) Breeding Environment has emerged to provide the necessary context for effective creation of dynamic virtual organizations (Camarinha-Matos, et al., 2009).

VO Breeding Environment (VBE) represents an association of organizations and their related supporting institutions, adhering to a basic long- term cooperation agreement, and adoption of common operating principles and infrastructures, with the main goal of increasing their preparedness towards rapid configuration of temporary alliances for collaboration in potential Vo. Namely, when one member (acting as a broker) identifies a business opportunity. Earlier cases of VBEs were mostly focused on a regional basis, e.g. industry clusters, industry districts and business ecosystem. Besides the production / services focus, a large number of more recent VBEs focus in on new areas, e.g. science and virtual laboratories, crises management etc. Simply put, network is comprised of a group of members linked to one another, including persons, teams, organizations, information and etc. Every organization interested in maintaining its' competitiveness should present in networks (Camarinha-Matos, et al., 2009). Table1 shows reasons to join VBE.

$\mathrm{CN}$ have shown that they have a high potential for creating an appropriate situation for innovations, application of technologies, satisfaction of unpredictable needs and maintaining the competitiveness. ACN includes independent members from different geographical locations that work under different conditions, have special cultures and social investment and define specific objectives for themselves and, finally, use IT and ICT for improving collaborative business. Depending on organizational structure, communication mechanisms which are an integrating part and also a method for exchanging information in dependent tasks or processes between members, have an important role in $\mathrm{CN}$. A CN is a virtual network for global production, made up of a group of active companies with fix and specific capabilities that maintain vertical and horizontal communication network between themselves, other independent companies and even their competitors. Unlike maintaining internal resources, mission of members in this network is to pay attentions to management and effective resource sharing.

As an organization which has various groups of entities, internal relationship in a $\mathrm{CN}$ should have enough flexibility (Camarinha-Matos, et al., 2009).

\section{Moving from GC to CN_GC:}

As it has been stated before due to the high level of uncertainty in construction projects, which is resulted from the

- Increase in activities/profit

- Coping with market turbulence

- Increasing chances of survival

- More chances to compete with larger companies

- Lobbying and market influence

- Easier access to loans

- Cheaper group insurance

- Better negotiation power

- Prestige, reputation, reference

- Access/explore new market/product

- Expand geographical coverage

- Increase potential for innovation

- Economy of scale

- Develop branding

- Achieve (global) diversity

Table 1. Examples of reasons to join a $\mathrm{VBE}^{1}$

${ }^{1}$ (Camarinha-Matos \& Afsarmanesh, 2006) 


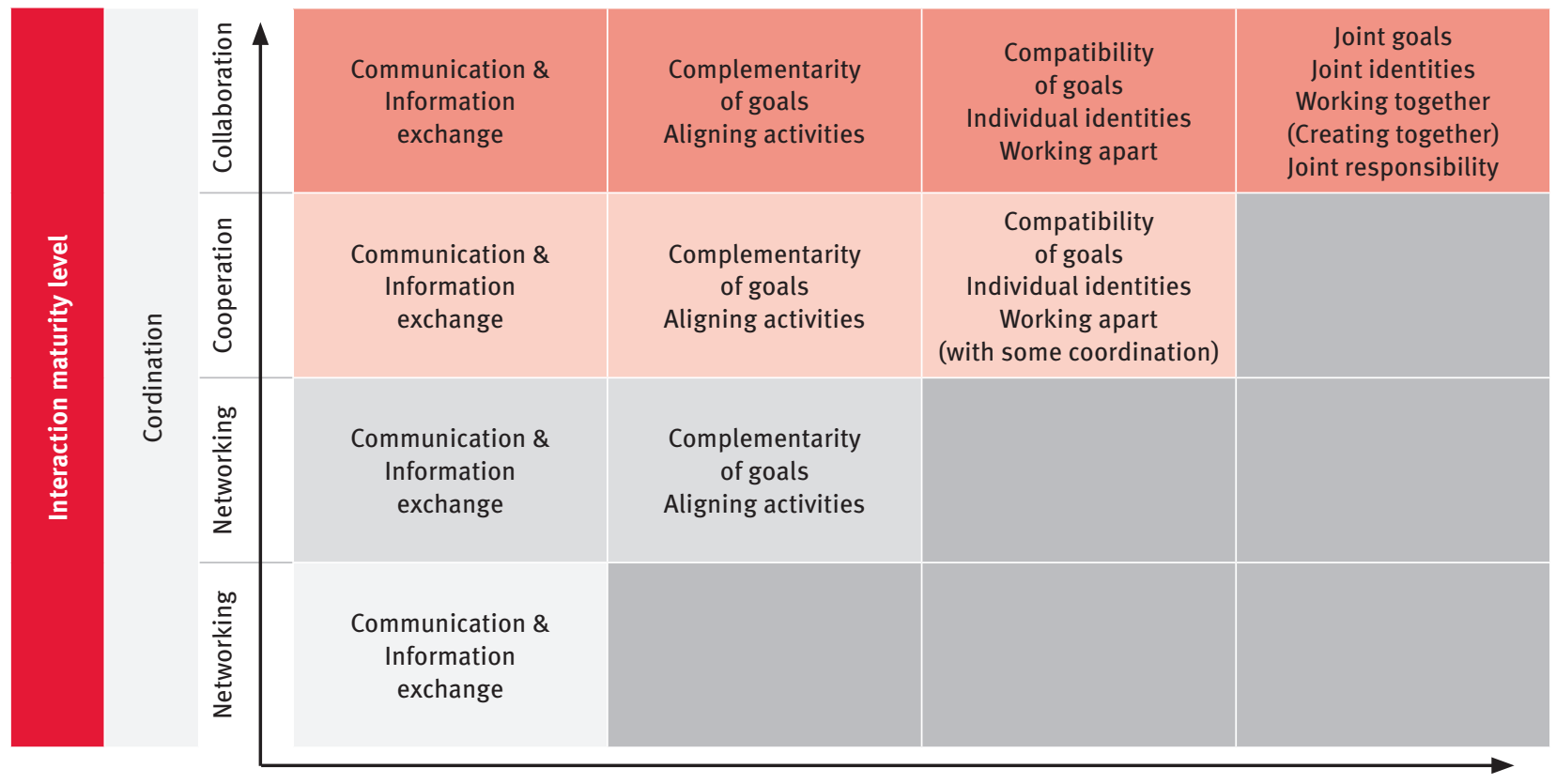

Integration Level

Figure 1. Interaction maturity levels

presence of various sub-contractors and suppliers, the general contractor should focus on coordination with subcontractors that is one of the most complex tasks in construction. To do this, GC need to use their resources, including: time, materials, and even labor.

Since construction projects cannot be completed by a single individual or organization, every project involves a close and complex collaboration of heterogeneous firms (Son \& Rojas, 2011).

\section{Providing a CO:}

A large variety of collaborative networks have emerged during the last years as a result of the challenges faced by both the business and scientific worlds (Camarinha-Matos \& Afsarmanesh, 2005). Figure 1 shows interaction maturity levels in enterprises. Networking involves communication and information exchange for mutual advantage. A simple example of networking is the case in which a group of entities share information about their experience by using a particular tool. Coordinated networking in addition to communication and information exchange involves aligning/altering activities, so that final results will be more efficient. Cooperation involves not only communication, information exchange, and adjustments of activities, but also resource sharing for realization of compatible goals. Division of some labor (not extensive) among participants also helps in achieving cooperation. Collaboration is a more demanding process in which entities share information, resources and responsibilities to jointly plan, implement, and evaluate a program of activities to achieve a common goal and therefore jointly generating value. This concept is derived from the Latin collaborate meaning "to work together" and can be seen as a process of shared creation; thus it is a process through which a group of entities enhance the capabilities of each other.

Coordination extends networking; cooperation extends coordination and collaboration extends cooperation. As we move along the continuum from networking to collaboration, we increase the amounts of common goal-oriented risk taking, commitment and resources that participants must invest into the joint endeavor (Camarinha-Matos \& Afsarmanesh, 2006)

The recent trend in enterprise collaboration is building partner relationship between organizations. Partner relationships are characterized by a hierarchical framework, such that an enterprise does not have total control over the others. Partnered enterprises achieve their goals only through the exchange of information, negotiation and coordination. The general purpose of enterprise collaboration is usually to maximize marginal profit or to hold the market punctuation along with minimizing negative impacts (Yoon, et al., 2011).

After reviewing both fields' (GC and $\mathrm{CN}$ ) literature, a comparison has been done to find common points. In Table 2 all the $\mathrm{GC}$ necessities and $\mathrm{CN}$ characteristics are seen; it is noticeable that establishing a $\mathrm{CN}$ will help in enhancing the role of GC from several aspects and it is possible because of the similarities existing between structure and characteristics of GC and CN. 
- Goal-oriented

- Network structure

- Sharing capabilities and resource

- Distributed Topology

- Members in both of them have specific resource and capabilities

- Members of both are experts and capable of planning

- Projects in GC are temporary and short-term but relations can be permanent.

- GC needs high financial competence and CN Increases activities and profit
- $\mathrm{CN}$ is a dynamic and open network but GC is static and closed - Members of GC are related to central core of companies and ordered based on a hierarchy but members of $\mathrm{CN}$ are autonomous and independent with holarchy structure - $\mathrm{GC}$ is a real network but $\mathrm{CN}$ is virtual

- Relations in GC are intra-enterprise but $\mathrm{CN}$ has inter-enterprise relations with internet infrastructure.

Table 2. Comparison between GC and CN

\section{Conclusion :}

Although this study reveals how new achievements of networks knowledge area particularly collaborative network concept can use to enhance capability of GC. Considering the similarity between advantages of implementation a CN with key performance indexes of $\mathrm{GC}$ leads us to make more researches to find feasibility of joining GC to kinds of CN. According to what has been discussed and reviewed, it is noticeable that establishing a $\mathrm{CN}$ will help in enhancing the role of $\mathrm{GC}$ from several aspects and it is possible because of the similarities existing between structure and characteristics of GC and CN. Furthermore, reviewing necessities of $\mathrm{GC}$ in a construction project with organizational achievements in $\mathrm{CN}$, we can have a prospective of future projects handled via new Generation of $\mathrm{GC}$ which are implemented in body of CNOs. Future competitive requirements of those SME organizations active in construction projects illustrate that, viability of these companies depends on robustness of their cooperative networks as well as relation maintained with other parties in this industry to use abilities and opportunities in order to survive and gain success.

Suggested topics for future research include lessons learned from manufacturing knowledge area for $\mathrm{CN}$ and defining critical success factors and key performance indexes for implementation of $\mathrm{CN}$ in construction projects. In addition, exploring the effect of absorptive capacity on the relationships between $\mathrm{CN}$ and $\mathrm{GC}$ performance by project size and type. Necessary contract strategies between $\mathrm{CN}$ parties to create a successful partnership area field to research.

\section{References:}

Ballard, G. \& Kim, Y.-W., 2005. Profit-point analysis: A tool for general contractors to measure and compare costs of management time expended on different subcontractors. Canadian Journal of Civil Engineering, pp. 712-718.

Camarinha-Matos, L. M. \& Afsarmanesh, H., 2006. A Modeling Framework for Collaborative Networked Organization. IFIP International Federation for Information Processing, pp. 25-27.

Camarinha-Matos, M. L. \& Afsarmanesh, H., 2005. Collaborative Networks: a new scientific discipline. Journal of Intelligent Manufacturing, Volume 16, p. 439-452.

Camarinha-Matos, M. L. \& Hamideh, A., 2006. Collaborative Networks, Value creation in a knowledge society. Proceedings of PROLAMAT, Volume 6, pp. 14-16.

Camarinha-Matos, M. L., Hamideh, A., Galeano, N. \& Molina, A., 2009. Collaborative Networked organizations - Concepts and practice in manufacturing enterprises. Computers \& Industrial Engineering, Volume 57, pp. 46-60. 
Chinowsky, P., Diekmann, J. \& Galotti, V., 2008. Social Network Model of Construction. Journal Of Construction Engineering And Management, pp. 804-812.

Dorogovtsev, S. N., Mendes, J. F. \& Samukhin, A. N., 2003. Metric structure of random networks. Nucl. Phys., Volume 653, p. 307-338.

Fan, C. \& Meng-jun, W., 2008. Research on Subcontracting Boundary of Engineering Project.. IEEE, pp. 1-4.

Han, S. H., Kima, D. Y., Jang, H. S. \& Seokjin, 2010. Strategies for contractors to sustain growth in the global construction market. Habitat International, Volume 34, pp. 1-10.

Hyun-soo Lee, Joon-oh Seo, Moonseo Park, Han-guk Ryu, Soon-seok Kwon, 2009.

Transaction-Cost-Based Selection

of Appropriate General ContractorSubcontractor Relationship Type. Journal of Construction Engineering and Management, pp. 1232-1240.

PARK, H. \& HAN, S. H., 2012. Impact of InterFirm Collaboration Networks in International Construction Projects: A Longitudinal Study. s.l., ASCE, pp. 140-1470.

Schoenmakers, W. \& Duysters, G., 2006. Learning in strategic technology alliances. Technology Analysis \& StrategicManagement, Volume 18, pp. 245-264.

Shuquan, L. \& Kongguo, Z., 2008. Research on Multi-Objective Optimization ofConstruction Project. s.l., s.n., pp. 480-483.

Son, J. \& Rojas, E. M., 2011. Evolution ofcollaboration in temporary project teams: an agent-based modeling and simulation approach. $137(8)$, pp. 619-628.

Tserng, P. H. \& Yin, S. Y. L., 2010. Developing an ARIS-House-Based Method from Existing Information Systems to ProjectBased Enterprise Resource Planning for General Contractor. Journal Of Construction Engineering And Management, pp. 199-209.

Wen, C. \& Xiao, M., 2010. Studying the Development Method of General Contractor of China. IEEE, pp. 1-4.

Xiaolin, Y., Heng, S. \& Bing, Y., 2008. A Supplier Selection Method for General ContractorBased on Benchmark Thinking and Case-Based Reasoning. IEEE, pp. 1-4.

Yoon, S. W., Matsui , M., Yamada, T. \& Nof, S. Y., 2011. Analysis of effectiveness and benefits of collaboration modes with information- and knowledge-sharing

Journal of Intelligent Manufacturing, 22(1),

pp. 101-112.

Yoon, W. S. \& Nof, Y. S., 2011. Affiliation/ dissociation decision models in demand and capacity sharing collaborative network. Int. J. Production Economics, Volume 130, pp. $135-143$.

Zippiness, C. \& Emmerich, W., 2008. A reference model of virtual service production networks. SOCA, Volume 2, p. $145-166$. 